\title{
METHOTREXATE-INDUCED ACCELERATED NODULOSIS: CAN IT OCCUR IN ANY ORGAN?
}

Marcus Villander Barros de Oliveira Sá1,2,3*, Leonardo José de Cupertino Barreto da Rocha Andrade ${ }^{3}$. Nara Gualberto Cavalcanti ${ }^{4}$, Daniela Mayumi Takano 1,4, Francisco Aristofanes Coelho Sarmento Neto', José Fernando do Prado Moura', Francisco José Trindade Barretto', Gustavo Henrique de Sá Miranda Cavalcante Filho', Flávio José Siqueira Pacheco', Jorge Luiz Carvalho Figueredo', Luydson Richardson Silva Vasconcelos ${ }^{2,3}$

1. Real Hospital Português de Beneficência em Pernambuco, Recife (PE), Brazil. 2. Instituto Aggeu Magalhães, Recife (PE), Brazil. 3. Universidade de Pernambuco, Recife (PE), Brazil. 4. Universidade Federal de Pernambuco, Recife (PE), Brazil.

*Corresponding author: marcusvillander@yahoo.com.br

\section{BACKGROUND}

Rheumatoid nodulosis is the most common extra-articular manifestation of rheumatoid arthritis (RA), with a prevalence of 10 to $30 \%$. In less than $1 \%$ of cases, they can occur systemically (noncutaneously), affecting sites such as lungs, heart and central nervous system.

\section{CASE REPORT}

We describe the case of a 56 -year-old man using methotrexate ( $20 \mathrm{mg} /$ day) and prednisone $(12 \mathrm{mg} /$ day) for seronegative rheumatoid arthritis for three years who sought medical attention for left low back pain without other associated symptoms. Abdominal CT showed ureterolithiasis on the left side, but the findings that drew attention were multiple pulmonary, hepatic and adrenal nodules, the largest with 2.5, 3.9 and $2.4 \mathrm{~cm}$, respectively (Fig. 1A and 1B; red and yellow arrows). There was no report of fever, weight loss, dyspnea, chest pain, cough, jaundice or other symptoms. Measurement of tumor markers (CA 19.9, CA 72.4 and CEA, alpha-fetoprotein, beta-HCG, thyroglobulin), serologies for HIV, hepatitis B and C were negative. Screening of neoplasms by upper gastrointestinal endoscopy, colonoscopy, thyroid function, breast and scrotal ultrasound and mammograms were negative. Serum RF, anti-CCP, antinuclear factor, antineutrophil antibody, complement dosage (C3, C4, CH50), lgG4 and angiotensin-converting enzyme levels were also normal. A CT-guided aspiration biopsy of pulmonary and hepatic nodules showed a necrotic nodule surrounded by lymphohistiocytic infiltrate (Fig. 2), a finding confirmed by immunohistochemistry. Ziehl-Neelsen staining for mycobacterial examination and fungus staining by PAS were negative. Cultures for Mycobacterium in LowensteinJensen and Stonebrink solid media and in Middlebrook 7H9 + OADC (Bactec) both were negative too. Mycobacterial DNA detection by GeneXpert was also negative. We chose to suspend the use of MTX. Two months after withdrawal of MTX, there was a significant reduction in the number and dimensions of lesions. These findings were consistent with methotrexate-induced accelerate nodulosis in the lungs, liver and adrenal glands in a patient with seronegative rheumatoid arthritis. The presence of rheumatoid nodules in the lungs, although rare, is well documented. The finding of rheumatoid nodules in the liver and adrenal glands is exceptional. To date there are only two other reports of rheumatoid nodulosis in the liver and no case described in the adrenal gland. After 1 year, all nodules have vanished.

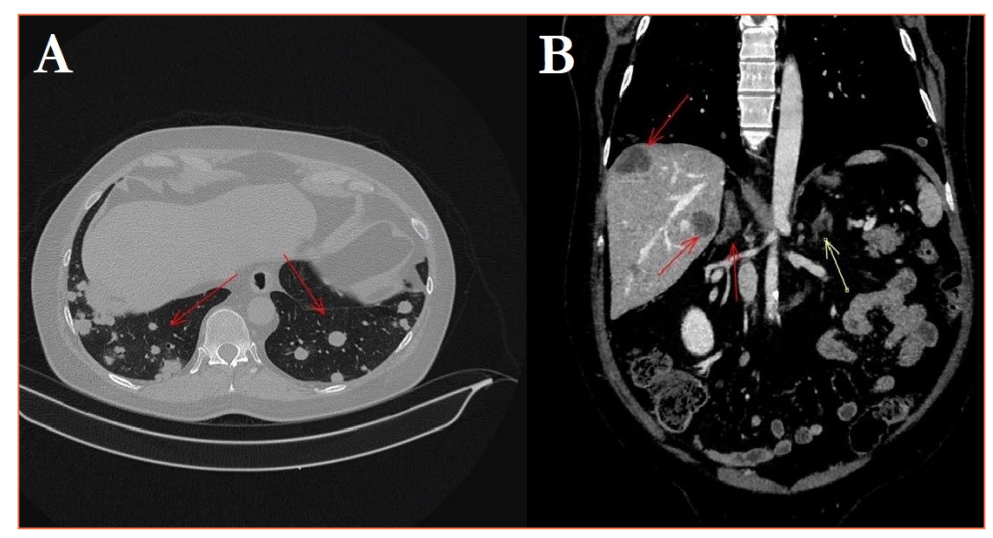

Figure 1. Abdominal computed tomography. 


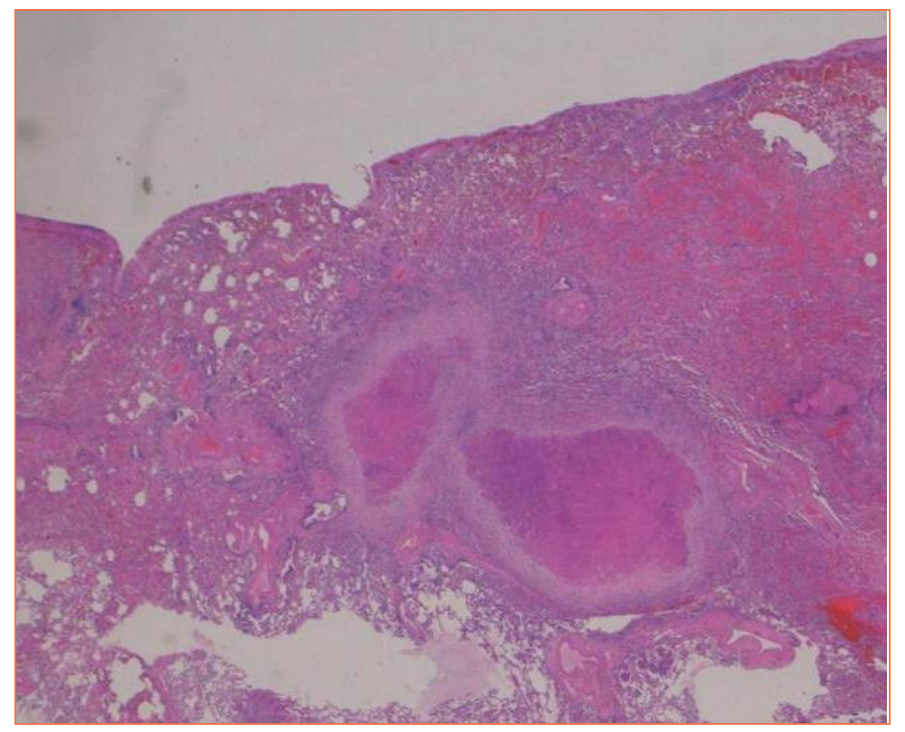

Figure 2. Computed tomography-guided aspiration biopsy of pulmonary and hepatic nodules.

\section{CONCLUSION}

We infer rheumatoid nodulosis can occur, virtually, in any organ and must be remembered in RA patients using methotrexate, regardless of the presentation of symptoms. 